\title{
Presence of conspecific females motivates egg cannibalism owing to lower risk of filial cannibalism
}

\begin{abstract}
A cannibal has to weigh the benefits of the consumption and removal of competing conspecifics against the potential loss of fitness through filial cannibalism. We examined the role of the presence of conspecific females in informing adaptive cannibalism decisions. Females of the hemipteran bug Geocoris pallens express low egg cannibalism when alone but become much more cannibalistic in the presence of conspecific females and do not discriminate between their own eggs and those of other females. Experimentation showed that females that could not commit filial cannibalism exhibited strong egg cannibalism that was not reduced by the presence of conspecific females, whereas females that could commit filial cannibalism were very cannibalistic only in the presence of conspecifics. An experiment also showed that the presence of conspecific females triggered a stronger egg cannibalism response in G. pallens than did a heterospecific egg predator. These results suggest that G. pallens females become cannibalistic in the presence of conspecifics because they interpret conspecific presence primarily as an indication of decreased likelihood of committing filial cannibalism, and less so as an indication of lower expected survival of eggs or future resource competition. Our study highlights the importance of informational cues, in this case the presence of conspecifics, in modulating the expression of cannibalism.
\end{abstract}

Keyword: Adaptive risk management; Context-based cue; Density-dependent cannibalism; Geocoris pallens; Kin discrimination; Predation risk; Size-structured interaction 